\title{
Multiple Scattering Theory of Spin-Polarized X-ray Photoelectron Diffraction*
}

\author{
Kouta Ito ${ }^{\dagger}$ Hiroshi Shinotsuka, Takehisa Konishi, and Takashi Fujikawa \\ Graduate School of Science, Chiba University, Yayoi-cho 1-33, Inage, Chiba 263-8522, Japan
}

(Received 14 October 2005; Accepted 8 November 2005; Published 29 November 2005)

\begin{abstract}
Spin-polarized X-ray photoelectron diffraction (SPXPD) spectra are useful to study magnetic structures of solid surfaces. In this work two different SPXPD modes are studied on the basis of a relativistic quantum electrodynamic (QED) theory which provides a unified picture of the SPXPD analyses. An important advantage of this theory is to allow us to handle many-body relativistic XPS theory, which can be a basis of SPXPD analyses. One mode is to utilize the spin polarization caused by multiplet splittings as observed in $\mathrm{O}_{2}$ molecules and Gd metals. We demonstrate how to extract local magnetic order in surface $\mathrm{O}_{2}$. The second SPXPD mode is to use circularly polarized X-rays to discriminate the spin polarization with aid of spin-orbit coupling. [DOI: 10.1380/ejssnt.2005.353]
\end{abstract}

Keywords: relativistic QED theory; multiple scattering theory; multiplet splitting; spin-polarized x-ray photoelectron diffraction (SPXPD)

\section{INTRODUCTION}

Spin polarization in the outermost surface layer can be different from that in the bulk.[1] Among several tools for the study of surface magnetic structures, spinpolarized x-ray photoelectron diffraction (SPXPD) is one of promising methods because it directly provides information about important local surface magnetic structures. Here SPXPD is studied on the basis of multiple scattering theory in the nonrelativistic and relativistic theoretical framework. The latter approach is inevitable to study XPD spectra from heavy elements.[2] XPD spectra give us information on structures at surface, while SPXPD spectra give us information on magnetic structures at surface.

SPXPD spectra are classified into two different modes. One mode is to utilize the spin polarization caused by multiplet splittings as observed in $\mathrm{O}_{2}$ molecules and $\mathrm{Gd}$ metals. If we use the multiplet splittings in $\mathrm{O} 1 \mathrm{~s}[3]$ or $\mathrm{Gd}$ $4 \mathrm{~s}, 5 \mathrm{~s}$ photoemission, $[4]$ we can detect spin-polarized photoelectrons excited by conventional X-ray sources without use of synchrotron radiation. We thus expect that $\mathrm{O} 1 \mathrm{~s}$ SPXPD spectra from solid $\mathrm{O}_{2}$ surface could provide some useful information about magnetic order.

The second SPXPD mode is to use circularly polarized Xrays to induce the spin polarization of photoelectron with aid of spin-orbit coupling. [5] This method is restricted to the photoemission from rather heavy elements in which LS couplings play important roles.

So far a general one-electron relativistic SPXPD theory has been developed by the present authors on the basis of multiple scattering theory. [2] This theory can describe the second mode of SPXPS, whereas the first mode is out of the scope because the multiplet splittings are basically a many-body effect in the core-hole states. We thus develop a many-body SPXPD theory based on a relativistic quantum electrodynamic (QED) XPS theory. [6] This theoretical framework provides a unified view of these two different SPXPD modes. As an example, we demonstrate

\footnotetext{
*This paper was presented at International Symposium on Surface Science and Nanotechnology (ISSS-4), Saitama, Japan, 14-17 November, 2005.

$\dagger$ Corresponding author: graduate.stereo3.chiba-u.jp@graduate. chiba-u.jp
}

a powerfulness of the SPXPS analyses for SPXPD of O1s photoemission from solid $\mathrm{O}_{2}$ surfaces.

\section{RELATIVISTIC QED SPIN-POLARIZED XPS THEORY}

To describe many-body spin-polarized XPS spectra, we have developed a relativistic QED Keldysh-Green's function approach, which provided a sound theoretical basis for the analyses. [6]

In the QED approach we can assume that the longitudinal one-electron Green's function $G_{L}(1,2)$ only depends on the time difference $t_{1}-t_{2}$, and we can define the spectral representation of $G_{L}(1,2)$. As the advanced and retarded Green's function $g^{a}$ and $g^{r}$ satisfy the closed Dyson equations

$$
\begin{gathered}
g^{r}(\varepsilon)=g_{0}^{r}(\varepsilon)+g_{0}^{r}(\varepsilon) \Sigma^{r}(\varepsilon) g^{r}(\varepsilon) \\
g^{a}(\varepsilon)=g_{0}^{a}(\varepsilon)+g_{0}^{a}(\varepsilon) \Sigma^{a}(\varepsilon) g^{a}(\varepsilon),
\end{gathered}
$$

these Green's functions are given in the energy space

$$
\begin{aligned}
& g(\varepsilon) \\
& =\left[\varepsilon+c^{2}-c \alpha \cdot \mathbf{p}-c^{2} \beta-V_{H}-\Sigma_{L}(\varepsilon)\right]^{-1} \\
& =\left(\begin{array}{lc}
\varepsilon-V_{H}-\Sigma_{11} & -c \sigma \cdot \mathbf{p}-\Sigma_{12} \\
-c \sigma \cdot \mathbf{p}-\Sigma_{21} & \varepsilon+2 c^{2}-V_{H}-\Sigma_{22}
\end{array}\right)
\end{aligned}
$$

where the Hartree potential $V_{H}$ is separated from the longitudial (electron-electron interaction) self-energy $\Sigma_{L}$ which has four components $\Sigma_{i j}(\mathrm{i}, \mathrm{j}=1,2)$. After some algebra we rewrite the relativistic retarded and advanced Green's function $g$ in terms of the nonrelativistic oneelectron Green's function $g_{11}$ and operator Q,

$$
\begin{array}{rlr}
g(\varepsilon) & =(1-X)^{-1}\left(\begin{array}{cl}
g_{11} & g_{11} Q \\
Q g_{11} & \\
Q g_{11} Q+\frac{1}{2 c^{2}}
\end{array}\right) \\
Q & =\frac{\sigma \cdot \mathbf{p}}{2 c}
\end{array}
$$

where the Gesztesy expansion [7] is extended to more complicated correlated Green's functions as shown by eq.(1): 
$X$ and its components are given by

$$
\begin{aligned}
X & =\left(\begin{array}{cc}
g_{11} Q \Sigma_{21} & X_{12} \\
X_{21} & X_{22}
\end{array}\right), \\
X_{12} & =g_{11}\left[\Sigma_{12}-Q\left(\varepsilon-V_{H}-\Sigma_{22}\right)\right] \\
X_{21} & =\left(Q g_{11}+\frac{1}{2 c^{2}}\right) \Sigma_{21} \\
X_{22} & =Q g_{11} \Sigma_{12}-\left(Q g_{11} Q+\frac{1}{2 c^{2}}\right)\left(\varepsilon-V_{H}-\Sigma_{22}\right) .
\end{aligned}
$$

The quasi-nonrelativistic one-electron Green's function $g_{11}$ satisfies the Dyson equation in the Keldysh contour for the selfenergy $\Sigma_{11}$

$$
\begin{gathered}
g_{11}(1,2)=g_{11}^{0}(1,2)+\int_{c} d 3 d 4 g_{11}^{0}(1,3) \Sigma_{11}(3,4) g_{11}(4,2), \\
g_{11}^{0}(\varepsilon)=\frac{1}{\varepsilon-T_{e}-V_{H}+i \eta} \quad, T_{e}=\frac{p^{2}}{2} .
\end{gathered}
$$

From eqs.(2) and (4), we obtain an approximate formula up to the relativistic order $g_{11}(\epsilon) / c^{2}$,

$$
\begin{aligned}
g(\epsilon) & =\left(\begin{array}{cc}
g_{11}+\delta g_{11}(\varepsilon) & g_{11} Q \\
Q g_{11} & Q g_{11} Q+\frac{1}{2 c^{2}}
\end{array}\right), \\
\delta g_{11}(\varepsilon) & =g_{11} Q \Sigma_{21} g_{11}+g_{11} \Sigma_{21} Q g_{11}+g_{11} Q\left(\Sigma_{22}+V_{H}-\epsilon\right) Q g_{11} .
\end{aligned}
$$

The relativistic GW approximation yeilds the selfenergy submatrix $[6]$

$$
\begin{aligned}
& \Sigma_{11}(1,2)=-i p_{1}\left[g_{11}(1,2)+\delta g_{11}(1,2)\right] W\left(2,1^{+}\right), \\
& \Sigma_{12}(1,2)=i p_{1} g_{11}(1,2) Q W\left(2,1^{+}\right), \\
& \Sigma_{21}(1,2)=i p_{1} Q g_{11}(1,2) W\left(2,1^{+}\right), \\
& \Sigma_{22}(1,2)=-i p_{1}\left[Q g_{11}(1,2) Q+\frac{1}{2 c^{2}}\right] W\left(2,1^{+}\right) .
\end{aligned}
$$

We notice that only $\Sigma_{11}$ has finite contribution even in the nonrelativistic limit $c \rightarrow \infty$.
The component $(\mathrm{i}=\mathrm{x}, \mathrm{y}, \mathrm{z})$ of total photoelectron current density induced by the X-ray field is given at detection position $\mathbf{r}$ and time $t$ by use of the Gordon relation [8] neglecting unimportant terms

$$
\begin{aligned}
j^{i}(\mathbf{r}, t) & =c \operatorname{Tr}<\psi^{+}(\mathbf{r}, t) \gamma^{0} \gamma^{i} \psi(\mathbf{r}, t)> \\
& \approx-\left.\frac{1}{2}\left(\nabla_{i}^{\prime}-\nabla_{i}\right) \operatorname{Tr}\left[g^{<}\left(\mathbf{r}^{\prime} t, \mathbf{r} t\right)\right]\right|_{\mathbf{r}^{\prime}=\mathbf{r} \rightarrow \infty}
\end{aligned}
$$

The lowest order Green's function $g^{<}$in regard to the electron-photon coupling term is explicitly given by [6]

$$
g^{<}(1,2) \sim 4 \pi i \int d 3 d 4 g^{r}(1,3) \gamma_{i} g^{<}(3,4) g^{a}(4,2) \gamma_{j} D^{>j i}(4,3),
$$

where $D^{>j i}$ is transverse photon Green's function. We apply the Gesztesy expansion (5) to $g^{r}$ and $g^{a}$ in eq.(8), which yields a useful formula of the photoelectron current with momentum $\mathbf{p}$, spin $\sigma$ and kinetic energy $\varepsilon_{p}\left(=p^{2} / 2\right)$ excited by $(\mathbf{k} s) \mathrm{X}$-ray photons

$$
\begin{array}{r}
\mathbf{j}_{\mathbf{p} \sigma} \propto \mathbf{p} \sum_{n}\left|S_{n}\right|^{2}\left[<f_{\mathbf{p} \sigma}^{-}|\triangle| \varphi_{c}><\varphi_{c}\left|\triangle^{*}\right| f_{\mathbf{p} \sigma}^{-}>\right. \\
+<f_{\mathbf{p} \sigma}^{-}|\triangle| \varphi_{c}><\varphi_{c}\left|\triangle^{*} g_{11}^{a} \delta T^{a}\right| f_{\mathbf{p} \sigma}^{-}> \\
+<f_{\mathbf{p} \sigma}^{-}\left|\delta T^{r} g_{11}^{r} \triangle\right| \varphi_{c}><\varphi_{c}\left|\triangle^{*}\right| f_{\mathbf{p} \sigma}^{-}> \\
+<f_{\mathbf{p} \sigma}^{-}|\triangle| \varphi_{c}><\chi_{c}\left|\triangle^{*} Q\right| f_{\mathbf{p} \sigma}^{-}> \\
+<f_{\mathbf{p} \sigma}^{-}|Q \triangle| \chi_{c}><\varphi_{c}\left|\triangle^{*}\right| f_{\mathbf{p} \sigma}^{-}> \\
+\cdots]<n_{\mathbf{k} s}>
\end{array}
$$

where $\left|\varphi_{c}\right\rangle$ and $\left|\chi_{c}\right\rangle$ are large and small components of the core function, $\mathbf{k}$ and $\mathbf{s}$ shows the $\mathrm{X}$-ray wave vector and its polarization, $\delta T^{r}$ and $\delta T^{a}$ include longitudinal relativistic effects on the photoelectron propagation in the targets,

$$
\begin{aligned}
& \delta T^{r}=Q \Sigma_{21}^{r}+\Sigma_{12}^{r} Q+Q\left(\Sigma_{22}^{r}+V_{H}-\varepsilon_{p}\right) Q \\
& \delta T^{a}=Q \Sigma_{21}^{a}+\Sigma_{12}^{a} Q+Q\left(\Sigma_{22}^{a}+V_{H}-\varepsilon_{p}\right) Q .
\end{aligned}
$$

The most important relativistic corrections are from oneelectron part $Q\left(V_{H}-\varepsilon_{p}\right) Q$ in eq.(10), and other relativistic corrections in eq.(10) are due to the many-body effects such as $Q \Sigma_{22} Q$, which should be much smaller than the above corrections. Equation (9) is thus rewritten in term of the nonrelativistic intrinsic amplitude $S_{n}=$ 
lation operator.

$$
\mathbf{j}_{\mathbf{p} \sigma} \propto \mathbf{p} \sum_{n}\left|S_{n}\right|^{2}\left|<f_{\mathbf{p} \sigma}^{-}\right| \triangle\left|\varphi_{c}>+<f_{\mathbf{p} \sigma}^{-}\right| \delta T^{r} g_{11}^{r} \triangle\left|\varphi_{c}>+<f_{\mathbf{p} \sigma}^{-}\right| Q \triangle\left|\chi_{c}>+\cdots\right|^{2}
$$

This formula is the basis for the SPXPD analyses. For $f_{\mathbf{p} \sigma}^{-}$we can apply site $\mathrm{T}$ matrix expansion, which yields multiple scattering series [2].

\section{SPIN-POLARIZED XPS FROM MULTIPLET-SPLITTING}

Now we try to apply the above theory to spin-polarized XPS spectra caused by multiplet-splittings. For example, $\mathrm{KMnF}_{3}$ gives considerably large energy splitting $(\sim 6.7 \mathrm{eV})$ in Mn 3s spectra.[9] This splitting is simply explained by the energy difference in the 3 s hole states, $3 s^{1} 3 d^{5}$. In the ${ }^{7} S$ final states, down-spin photoelectrons are excited, whereas in the ${ }^{5} S$ final states, up-spin photoelectrons are excited. In eq.(9) the amplitude $S_{n}$ reflects the difference in the final states. For the up-spin photoemission, the final hole state $|+\rangle$ is approximated by ${ }^{5} S$ atomic state in the case of weak ligand field theory.

Experimentally we observe energy splittings in Gd 4s $(\sim 4 \mathrm{eV}), 5 \mathrm{~s}(7 \sim 8 \mathrm{eV})$ in Gd metals [4] and O1s spectra $(\sim 1.12 \mathrm{eV})$ in $\mathrm{O}_{2}$ molecules.[3] Therefore we can obtain SPXPD spectra of the surface of Gd solid and oxygen solid, without measuring electron spins.

As an example, we study O1s SPXPD from antiferromagnetic solid oxygen at low temperature. For these systems the relativistic effects play a minor role, but eq.(9) provides unified view for photoemission from light and heavy elements. Figure 1 shows the multiplet splitting of

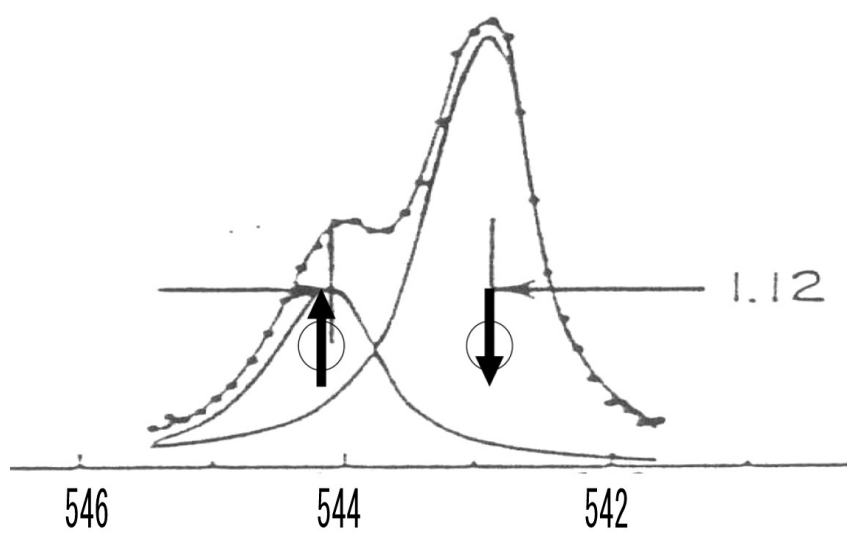

Binding energy $(\mathrm{eV})$

FIG. 1: Multiplet splitting of O1s XPS in free $\mathrm{O}_{2}$ molecules and spin polarization of photoelectrons. We can see that up spin photoelectrons and down spin photoelectrons have different binding energies. [3] (a)

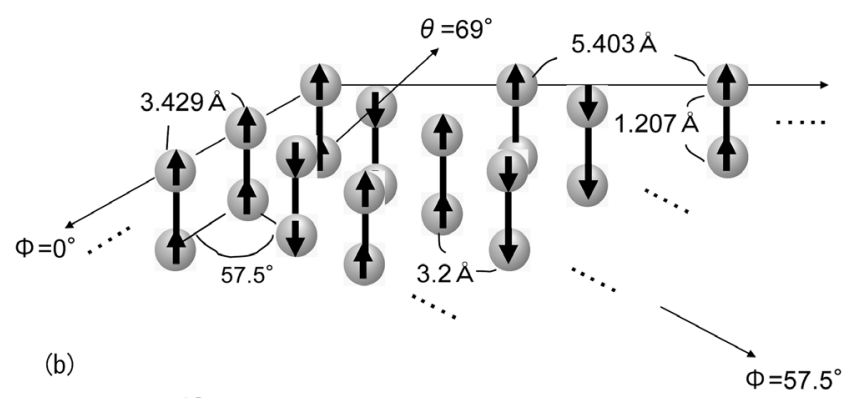

antiferro

$\theta=0^{\circ} \quad \theta=69^{\circ}$

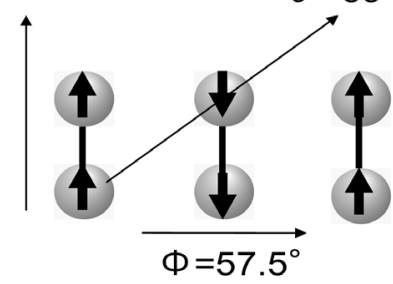

ferro

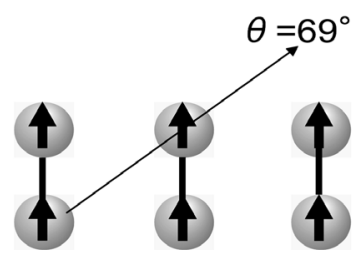

FIG. 2: (a)Surface structure of oxygen solid in $\alpha$-phase.[10] (b)Two layer models of the antiferromagnetic and artificial ferromagnetic spin orders are considered here.

O1s XPS. We can see that up spin photoelectrons have smaller kinetic energy than down spin photoelectrons. We apply the multiple scattering SPXPD calculations to $\mathrm{O}_{2}$ solid which is in antiferromagnetic $\alpha$ phase.[10] Figure 3 shows O1s SPXPD spectra for $\mathrm{O}_{2}$ solids calculated by multiple scatterings XPD theory given in the previous section. Here, we difine the spin polarization $P$ as

$$
P=\left[I_{\uparrow}\left(\mathbf{k}_{\uparrow}\right)-I_{\downarrow}\left(\mathbf{k}_{\downarrow}\right)\right] / \rho_{c}(1)^{2} .
$$

where $\rho_{c}(1)$ is the dipole radial integral between the core O1s function and photoelectron p wave, $I_{\uparrow}\left(\mathbf{k}_{\uparrow}\right)$ and $I_{\downarrow}\left(\mathbf{k}_{\downarrow}\right)$ are XPD intensities of up spin (at higher binding energy peak) and down spin. For the final doublet(up spin) and quartet(down spin) states, we should take the statistical weights 1 and 2 into account to compare the calculated intensities with the observed ones. To enhance the spin dependent scatterings, we rather use eq.(12) without the statistical weight. The calculated results for the polarization $P$ are shown in Fig.3 (a)-(c) for different X-ray 
energies 590, 1140, $1420 \mathrm{eV}$, together with the difference $\Delta P$ of $P$ 's for antiferromagnetic and artificial ferromagnetic models shown in Fig.2(b);

$$
\Delta P=P(\text { antiferro })-P(\text { ferro }) .
$$

In the case of excitation by the low energy photons, the spin polarization $P$ is large $(\sim 0.5 \%$ for $\omega=590 \mathrm{eV})$ relative to the $P$ excited by the high energy photons $(\sim 0.3 \%$ for $\omega=1420 \mathrm{eV})$. At the polar angle $\theta=69^{\circ}$ as shown in Fig.2(b), the forward spin-dependent scatterings can give rise to the defference between ferromagnetic and antiferromagnetic spin orders. The three SPXPD spectra excited by the three photon energies actually show small but detectable difference $\Delta P$ at that angle in the order of XMCD $(\sim 0.1 \%)$. The excitation by the low energy photons $(590 \mathrm{eV})$, however, gives the larger difference $\Delta P$ at lower angle $\left(\sim 60^{\circ}\right)$.

\section{SPIN-POLARIZED XPS BY USE OF CIRCULARLY POLARIZED X-RAYS}

Detailed one-electron relativistic theory has already been given in ref. [2]. In contrast to the one-electron theory the present many-body theory shows the amplitudes in terms of the damping photoelectron wavefunction $f_{\mathbf{p} \sigma}^{-}$ under the influence of an advanced self-energy (optical potential) $\Sigma^{a}\left(\varepsilon_{p}\right)$.[11] Some sophisticated practical approaches have been developed for the optical potential calculations. [12, 13]

In this section we discuss a spin-polarized XPD theory excited by circularly polarized X-rays. For this purpose eq.(11) also plays a central role. The SPXPD excited by circularly polarized X-rays from K-edge is quite different from $L_{2,3}$-edge excitation, since the spin-orbit coupling only works for photoelectrons in the former whereas it dominantly contributes to the splitting of $2 \mathrm{p}$ level to $L_{2}$ and $L_{3}$ levels. When the many-body effects in $\delta T^{r}$ are neglected, it is simply written by

$$
\begin{aligned}
& \delta T^{r} \sim Q\left(V_{H}-\varepsilon_{p}\right) Q \\
= & -\frac{1}{(2 c)^{2}}\left[\nabla V_{H} \cdot \nabla+\left(V_{H}-\varepsilon\right) \nabla^{2}\right]+\zeta(r) \sigma \cdot \mathbf{L}
\end{aligned}
$$

where $\zeta(r)$ is related to the spherically symmetric potential $v_{A}$ at X-ray absorbing site $\mathrm{A}\left(V_{H}+\Sigma^{a}\left(\varepsilon_{p}\right)=\right.$ $\left.v_{A}+\sum_{\alpha} v_{\alpha}\right)$

$$
\zeta(r)=\frac{1}{(2 c)^{2} r} \frac{d v_{A}(r)}{d r} .
$$

This operator $\delta T^{r}$ acts on photoelectron wave functions, so that the influence is quite small.

We should note that an important many-body effect, optical potential, is already included in $f_{\mathbf{p} \sigma}^{-}$and $g_{11}^{r}$ (see eq.(11)) in the present theory. The second term in eq.(11) is crucial for the spin polarization of photoelectron excited from K-edges by circularly polarized X-rays. [2]

In the case of $L_{2,3}$ edge excitation, the first term of eq.(11) dominantly contributes to the spin polarization excited by circularly polarized X-rays. Only the optical potential is the new factor to the previous one-electron SPXPD theory in this case.[2] (a)

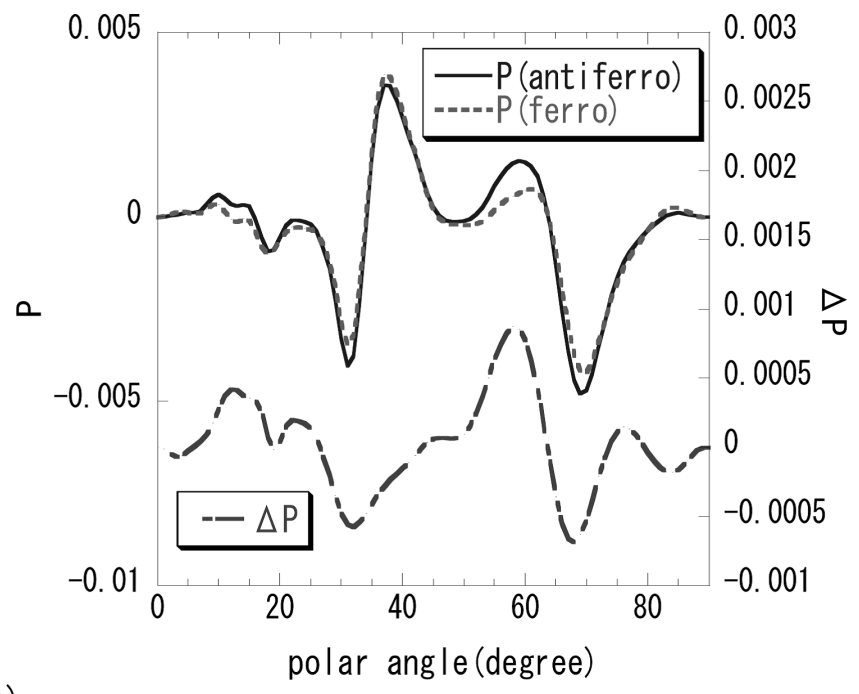

(b)

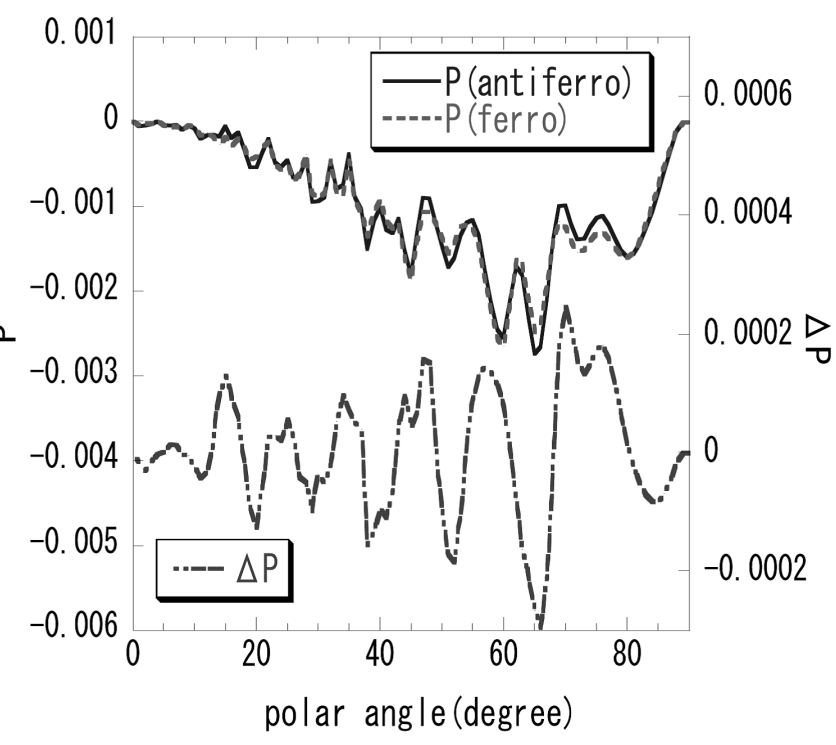

(c)

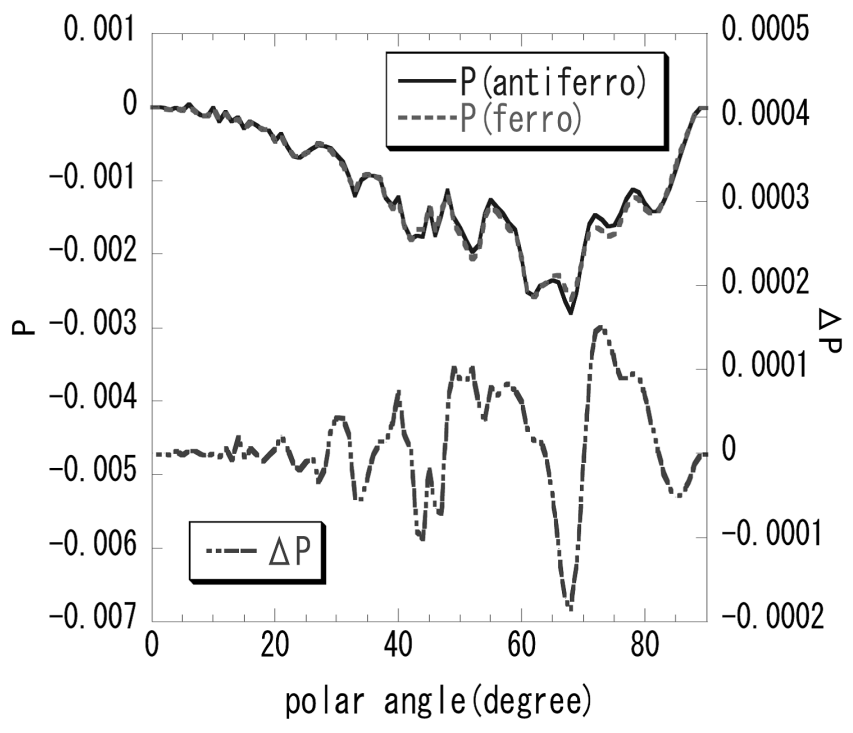

FIG. 3: The polar angle scans of the calculated O1s SPXPD for the two models shown in Fig.2 excited by (a) $590 \mathrm{eV}$, (b) $1140 \mathrm{eV}$ and (c) $1420 \mathrm{eV}$ X-ray photons. The spin polarization $P$ and the polarization difference $\Delta P$ are defined by eqs.(12) and (13). 


\section{CONCLUDING REMARKS}

QED theory provides basic theoretical framework to study SPXPD spectra, where relativistic effects play an important role. Two different types of SPXPD are discussed based on multiple scattering approaches. Both of the SPXPD analyses give us useful information about local spin ordering at solid surfaces even for antiferromagnetic systems, which can be an advantage over the X-ray magnetic circular dichroism (XMCD). The latter can be applied only to ferromagnetic systems. The present theory gives us unified view on these SPXPD spectra.
[1] R. Feder(ed), Polarized Electrons In Surface Physics, (World Scientific, 1985).

[2] T. Fujikawa and T. Konishi, J. Phys. Soc. Jpn. 72, 2265 (2003).

[3] D. W. Davis, R. L. Martin, M. S. Banna,and D. A. Shirley, J. Chem. Phys. 59, 4235 (1973).

[4] E. D. Tober, F. J. Palomares, R. X. Ynzunza, R. Denecke, J. Morais, Z. Wang, G. Bino, J.Liesegang, Z. Hussain, and C. S. Fadley, Phys. Rev. Lett. 81, 2360 (1998).

[5] K. Starke, Magnetic Dichroism in Core-Level Photoemission, (Springer, 2000).

[6] T. Fujikawa, J. Elect. Spect. Relat. Phenom. 136, 85 (2004).
[7] F. Gesztesy, H. Grosse and B. Thaller, Ann. Inst. Henri Poincare 40, 159 (1984).

[8] W. Greiner, Relativistic Quantum Mechanics, (Springer, 1990).

[9] B. Sinkovic and C. S. Fadley, Phys. Rev. B 31, 4665 (1985).

[10] N. Suzuki, M. Ootani, Polyhedron. 20, 1381 (2001).

[11] H. Arai and T. Fujikawa, Phys. Rev. B 72, 075102 (2005).

[12] T. Fujikawa, A. Saito and L. Hedin, Jpn. J. Appl. Phys. Suppl. 32-2, 18 (1993).

[13] T. Fujikawa, K. Hatada and L. Hedin, Phys. Rev. B 62, 5387 (2000). 